\title{
Analysis of the Effect of Soil Roughness in the Forward-Scattering Interference Pattern Using Second-Order Small Perturbation Method Simulations
}

\author{
Mariano Franco ${ }^{\circledR}$, Emanuel More, Esteban Roitberg ${ }^{\circledR}$, Francisco Grings ${ }^{\circledR}$, Member, IEEE, \\ Estefanía Piegari, Vanesa Douna, and Pablo Perna
}

\begin{abstract}
Soil moisture (SM) is a key geophysical variable that can be estimated at regional scales using remote sensing techniques, by making use of the known relationship between soil reflectivity and the dielectric constant in the microwave regime. In this context, the exploitation of available illuminators of opportunity that currently emit large amounts of power at microwave frequencies (compared to typical synthetic aperture radar systems) is promising. Some published techniques estimate SM by analyzing the interference pattern (IP) between direct and reflected signal as measured by a single antenna (i.e., IP technique). In this letter, a new approach to simulate the IP is proposed, in which the soil roughness is modeled straightforwardly using the second-order small perturbation model. Results illustrate that the "notch" in the VV-polarization IP (related to the Brewster angle) can only be directly observed for very low values of soil rms roughness $(s<0.5 \mathrm{~cm})$. For typical values of soil roughness $(s \sim 1.2 \mathrm{~cm})$, the notch disappears and only a minimum in the IP is observed near the Brewster angle.
\end{abstract}

Index Terms-Electromagnetic and remote sensing, Global Navigation Satellite System data, microwave radiometry, surface and subsurface properties.

\section{INTRODUCTION}

A TYPICAL approach for estimating surface soil moisture (SM) at regional scales and high spatial resolution is based on the exploitation of the signal reflected from the soil in the microwave regime, using the well-known relationship between the dielectric constant and SM. However, the scattered signal by the surface is not only determined by SM but also surface properties as its rms height $(s)$ and the geometry of the incident wave. Then, in order to get a retrieval algorithm for the SM, it is essential to count with a coherent scattering model which physically relates the relevant parameters involved in the scattering process. A well-established theoretical model to relate the soil backscattering coefficient $\left(\sigma^{0}\right)$ with the soil dielectric constant $(\epsilon)$ and soil rms roughness $s$ is the small

Manuscript received July 23, 2018; revised October 22, 2018; accepted November 18, 2018. This work was supported by PICT Raíces 2014-0647. (Corresponding author: Mariano Franco.)

M. Franco, E. Roitberg, F. Grings, E. Piegari, V. Douna, and P. Perna are with the Instituto de Astronomía y Física del Espacio (CONICET-UBA), Pabellón IAFE, CABA, Buenos Aires 1428, Argentina (e-mail: mfranco@df.uba.ar; verderis@iafe.uba.ar).

E. More is with the Instituto de Altos Estudios Espaciales "Mario Gulich," CONAE, Córdoba 5187, Argentina.

Digital Object Identifier 10.1109/LGRS.2018.2882688 perturbation model (SPM) [1], [2], which has been successfully studied under several different conditions for the surface or the incident wave [3]-[6]. However, to directly invert the second-order SPM (SPM-2) in order to estimate $\epsilon, \sigma^{0}$ of the terrain at high resolution is required, a task that can only be accomplished by a synthetic aperture radar, a relatively expensive, and power-demanding instrument.

In this letter, illuminators of opportunity (IOO) present several operative advantages for estimating SM. First, IOO bistatic radar configurations do not require a dedicated transmitter, which significantly reduces implementation costs (in both power and overall mass). Second, at least at L-band $(\lambda \sim 25 \mathrm{~cm})$, there is evidence that soil forward scattering presents a similar sensitivity to SM than the backscattered signal [7]. Typical SM retrieval techniques based on IOOs rely on the measurement of the soil forward-scattering coefficient, which is related to SM through the dielectric constant. The main benefit of this approach is that the ratio between reflected and transmitted fields is a direct proxy of SM. However, this approach has several drawbacks. One of the most significant drawbacks is the very good antenna isolation required to separate direct and reflected components (reflected component is usually -10 to $-20 \mathrm{~dB}$ below direct component, see [8]-[11]). Therefore, a very low level of crosstalk between antennas can be tolerated.

As an alternative, for IOOs characterized by sufficiently long pulses, the interference pattern technique (IPT) was proposed [12]. This technique is based on measuring the vertically polarized component of the received signal at the antenna. This "vertically polarized interference pattern (IP)" is analyzed in order to find a minimum ("notch"), which according to theory should correspond to the Brewster angle, which is itself related to the surface dielectric constant and SM [12]. Therefore, this technique has the advantage of requiring only one antenna and thus it does not rely on signal separation, but on the signal coherent sum. This technique has been successfully implemented and validated using fieldwork in [8], [11], and [13].

However, the analysis presented in [13] and its subsequents [14]-[16] present a key limitation: they are based on a simplified model for soil specular scattering. This means that it is assumed that soil specular scattering can be modeled 
using a plane interface multiplied by a roughness term to analyze the relationship between the angle in which the notch is found and then the dielectric constant of the soil. Therefore, the position and the presence of the notch itself are based on a simplified scattering model, which does take into account soil roughness but does not include multiple scattering effects on the surface. Hence, this analysis could be improved by using a more advanced scattering model.

In this letter, we implemented the SPM-2 [2]-[4] for simulating the vertically polarized IP expected in the antenna as a function of both geometric and dielectric soil properties (soil dielectric constant and roughness). We developed a model that computes the coherent sum between the electric field scattered by the soil and the direct component emitted by the IOO as a function of surface characteristics, assuming incident circular polarization and received vertical polarization. In the analysis, we show that the SPM-2 predicts the notch for very smooth soils. In this condition, the notch expected monotonic behavior as a function of soil dielectric constant is also observed. However, the existence of the notch depends strongly on soil roughness due to multiple scattering processes that take place in the illuminated surface at this frequency.

This letter is organized as follows. In Section II, we introduce the scattering model and a solution for the proposed geometry. In Section III, we present key simulation results that show the expected vertically polarized IP for different soil conditions and system configurations. In particular, the position and amplitude of the ITP minimum are studied. Finally, in Section IV, we present some conclusions derived from the analysis.

\section{SCATtering Model: Second-ORdeR Small Perturbation Method}

The SPM is based on the hypothesis that soil surface has a small $\mathrm{rms}$ height $(s)$ with respect to the incident wavelength $(\lambda)$, specifically that $2 \pi s / \lambda \ll 0.3$ [1], [2]. Usually, the SPM is used even in the limit $s \sim 0.05 \lambda$, producing satisfactory results (see [3]-[6]). For our case $(\lambda=25 \mathrm{~cm})$, the condition to be satisfied is $s<1.25 \mathrm{~cm}$, which is a reasonable assumption since for bare agricultural soils, typical values of rms height are $s \sim 1 \mathrm{~cm} \mathrm{[17]-[22].} \mathrm{This} \mathrm{approach}$ is based on proposing that the scattered and transmitted fields above and below the surface satisfy the boundary conditions of the Maxwell equations. The scattered and transmitted fields are written as a power series expansion in terms of the surface height $z$, where each term accounts for different scattering mechanisms on the surface. The zeroth-order term shows the specular reflection, the first-order term gives a single scattering behavior, and the second order represents a multiplescattering process where the incident wave after the first interaction with the surface goes to a second point, reflects again and finally propagates to the free space. At this point, it is important to remark that at the second order, the SPM verifies energy conservation [23]. Thus, we are proposing a physically based scattering theory that includes both surface roughness and energy conservation effects.

We implemented the SPM up to the second order to compute the IP observed in the antenna. We deal with circular polarization (GPS systems); therefore, the incident wave and reflected wave (RV) can be decomposed in terms of horizontal and vertical polarizations. As the antenna only measures vertical polarization, we must compute the vertical polarization of the RV, which has two components: VV and HV. The first one is due to a vertical incident polarization which remains in the same polarization after it interacts with the surface; the latter is due to the cross polarization effect, by which a wave with horizontal polarization changes its state after it scatters with the surface. It is well known that the cross polarization is due to multiple scattering effects [2]-[5], and thus it requires the development of the SPM up to the second order.

Based on SPM, the scattered field is expressed as [2]-[4]

$$
\mathbf{E}_{s} \approx \int \frac{d^{2} k}{(2 \pi)^{2}} e^{\imath \mathbf{k} \cdot \mathbf{r}} \sum_{n}\left[\alpha^{(n)} \hat{h}_{s}+\beta^{(n)} \hat{v}_{s}\right]
$$

being $\hat{h}_{s}$ and $\hat{v}_{s}$ the horizontal and vertical polarizations of the scattered field. Up to the second order, the amplitude of the vertical polarization mode is

$$
\begin{aligned}
\beta(\mathbf{k}) \approx \beta^{(0)} \delta\left(\mathbf{k}-\mathbf{k}_{i}\right) & +\beta^{(1)} Z(\mathbf{k}) \\
& +\int d^{2} k^{\prime} \beta^{(2)}\left(\mathbf{k}, \mathbf{k}^{\prime}\right) Z\left(\mathbf{k}-\mathbf{k}^{\prime}\right)
\end{aligned}
$$

with a similar expression for the amplitude $\alpha$ (for a detailed development of these coefficients, see [2]-[4]). First- and second-order terms depend on the Fourier transform of the surface, $Z(\mathbf{k})$. We see that the second-order term shows a process of multiple scattering through the integration of an auxiliary mode in $Z$. We have a set of parameters $\left\{\alpha^{(n)}, \beta^{(n)}\right\}$ for the TE mode and a different set for the TM mode.

Considering that we are interested in finding the scattered field in vertical polarization from an incident wave with circular polarization, we must compute the dot product between the expression (1) and $\hat{v}_{s}$. Thus, we will have the VV contribution given by the set of TE and the HV contribution given by the set of TM. For simplicity, we call them $\xi^{(n)}$. In Section II-A, we will compute the IP due to the incident and scattered waves.

$$
\begin{aligned}
\left\langle E_{T} E_{T}^{*}\right\rangle= & \frac{1}{E_{i}^{2}}\left\langle\left(E_{i}+E_{S}\right) \cdot\left(E_{i}^{*}+E_{s}^{*}\right)\right\rangle \\
\left\langle E_{T} E_{T}^{*}\right\rangle \approx & \left\langle\left\{e^{\imath \mathbf{k}_{i} \cdot \mathbf{r}_{A}}+\int \frac{d^{2} k}{(2 \pi)^{2}} e^{l \mathbf{k} \cdot \mathbf{r}_{A}}\left[\xi^{(0)} \delta\left(\mathbf{k}-\mathbf{k}_{i}\right)+\xi^{(1)} Z(\mathbf{k})+\int \frac{d^{2} k^{\prime}}{(2 \pi)^{2}} \xi^{(2)}\left(\mathbf{k}, \mathbf{k}^{\prime}\right) Z\left(\mathbf{k}-\mathbf{k}^{\prime}\right)\right]\right\}\right. \\
& \left.\times\left\{e^{-l \mathbf{k}_{i} \cdot \mathbf{r}_{A}}+\int \frac{d^{2} k}{(2 \pi)^{2}} e^{-l \mathbf{k} \cdot \mathbf{r}_{A}}\left[\xi^{(0)} \delta\left(\mathbf{k}-\mathbf{k}_{i}\right)+\xi^{(1)} Z(\mathbf{k})+\int \frac{d^{2} k^{\prime}}{(2 \pi)^{2}} \xi^{(2)}\left(\mathbf{k}, \mathbf{k}^{\prime}\right) Z\left(\mathbf{k}-\mathbf{k}^{\prime}\right)\right]\right\}\right\rangle
\end{aligned}
$$




\section{A. Interference Pattern}

With the goal of computing the IP received by the antenna, we must compute the mean value of the total field measured by it. As we have a stochastic RV, due to the scattering from a random rough surface, the IP will depend on statistical properties of the surface (i.e., roughness spectrum $W(\mathbf{k})$, rms height $s$, and correlation length $l$ ). Therefore, we must compute the mean power of the total intensity normalized to the incident field amplitude, which is shown in (3) shown at the bottom of the previous page.

Here, $\mathbf{r}_{A}=\left(x_{A}, z_{A}\right)$ indicates the antenna position. To perform the mean value, we only need to know that the surface can be modeled as a stationary and isotropic random process, with a Gaussian distribution of heights and zero mean. This kind of stochastic process has the following properties: $\langle Z(\mathbf{k})\rangle=0$ and $\left\langle Z(\mathbf{k}) Z^{*}\left(\mathbf{k}^{\prime}\right)\right\rangle=W\left(\mathbf{k}-\mathbf{k}^{\prime}\right)$, where the last quantity is the roughness spectrum of the surface, which we consider Gaussian, namely, $W(\mathbf{k})=$ $\left(s^{2} l^{2} / 4 \pi\right) \exp \left[-l^{2} \mathbf{k}^{2} / 4\right]$. By using these properties, the intensity pattern that emerges from a straightforward but tedious calculation results in

$$
\begin{aligned}
\left\langle E_{T} E_{T}^{*}\right\rangle \approx & +2 \xi^{(0)} \cos \left(2 k_{s z} z_{A}\right)+\left[\xi^{(0)}\right]^{2} \\
& +2 \Re\left[e^{\imath 2 k_{s z} z A} \int d^{2} k^{\prime} \xi^{(2)} W\left(\mathbf{k}_{i}-\mathbf{k}^{\prime}\right)\right] \\
& +\left|\xi^{(1)}\left(\mathbf{k}_{i}\right)\right|^{2} \int d^{2} k^{\prime} W\left(\mathbf{k}^{\prime}\right) \\
& +2 \xi^{(0)} \int d^{2} k^{\prime} \Re\left[\xi^{(2)}\left(\mathbf{k}_{i}, \mathbf{k}^{\prime}\right) W\left(\mathbf{k}_{i}-\mathbf{k}^{\prime}\right)\right] .
\end{aligned}
$$

The first line shown in (4) is similar (but not exactly equal) to the expression derived in [13], where the power intensity is proportional to the Fresnel reflection coefficient (here noted by $\left.\xi^{(0)}\right)$ multiplied by a Gaussian roughness factor and to the phase difference between the incident and RV (i.e., $\Delta \phi=$ $\left.2 k_{s z} z_{A}\right)$

$$
\begin{aligned}
\left\langle E_{T} E_{T}^{*}\right\rangle & \approx 1+2 \xi^{(0)} \cos \left(2 k_{s z} z_{A}\right)+\left[\xi^{(0)}\right]^{2} \\
& =\left|1+\xi^{(0)} e^{t 2 k_{s z} z_{A}}\right|^{2} .
\end{aligned}
$$

As we are using a scattering method based on small perturbations, the above-mentioned term does not include any roughness factor, because it is the zeroth-order solution. The surface roughness appears naturally in the following terms, which are proportional to the roughness spectrum $W(\mathbf{k})$. Moreover, for typical agricultural soils $(s \approx 1 \mathrm{~cm})$, both terms, $\xi^{(1)}$ and $\xi^{(2)}$, are relevant because they take into account the multiple scattering effects produced on the surface.

In Section III, we use (4) to simulate the behavior of the IP measured in the antenna.

\section{Simulated Results}

In Section II, we obtained the theoretical expression of the IP, which depends on the illumination geometry and surface parameters (geometric and dielectric). In Fig. 1, the behavior of the signal measured in the antenna is shown as a function of the incidence angle for several values of soil rms height $s$ : values near $1 \mathrm{~cm}$ are typical for no-till soil management [18], [19], [22]; other less typical management

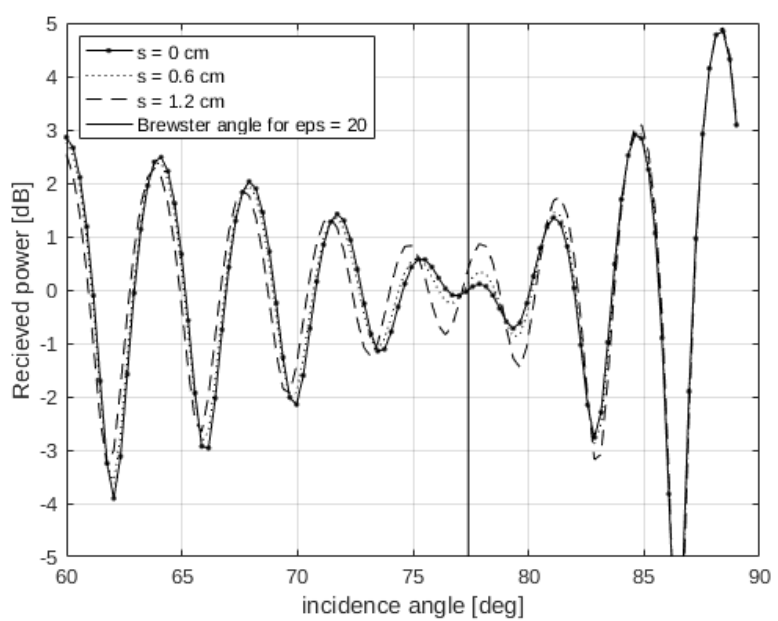

Fig. 1. Simulated IP as a function of the incidence angle for different values of the surface rms height $s$. Results correspond to L-band, i.e., $\lambda=25 \mathrm{~cm}$, correlation length $l=10 \mathrm{~cm}$, dielectric constant $\epsilon=20$ (which is related to a medium SM value), and the scattered angle is equal to the incidence angle $\theta_{s}=\theta_{i}$.

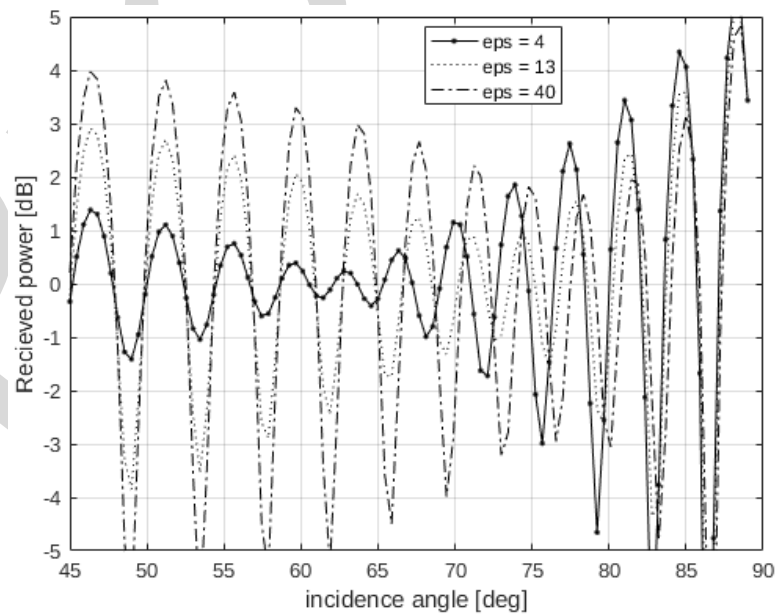

Fig. 2. Simulated IP as a function of the incident angle for different values of soils dielectric constant $\epsilon$. Results correspond to soil roughness $s=1 \mathrm{~cm}$ (typical of agricultural soils), correlation length $l=10 \mathrm{~cm}$, and L-band operating frequency. The scattered angle is equal to the incidence angle $\theta_{s}=\theta_{i}$.

(e.g., harrow or roll) lead to values $\sim 0.5 \mathrm{~cm} \mathrm{[20],} \mathrm{[21].}$ In addition, in plowed fields, the soil rms height can reach values in the range of $2.5-4 \mathrm{~cm} \mathrm{[20],} \mathrm{[21],} \mathrm{for} \mathrm{which} \mathrm{notch}$ detection will be more difficult, as we will show in Fig. 1. Finally, in order to plot the IP, the antenna is located at $z_{A}=2 \mathrm{~m}$ (or $8 \lambda$ in the worst case, which satisfies the far-field condition).

As expected for a flat surface $(s=0 \mathrm{~cm})$, a notch corresponding to the Brewster angle is present for large values of the incidence angle, which is in agreement with (5) and the results presented in [13]. As $s$ increases the notch starts to vanish, almost disappearing for relatively large values of the roughness $(s=1.2 \mathrm{~cm} \sim 0.05 \lambda)$. This is related to the fact that for a very rough surface, the diffuse scattering (proportional to the factors $\xi^{(1)}$ and $\xi^{(2)}$, which take into account the effects of multiple scattering) becomes more relevant than the specular reflection (just proportional to $\left.\xi^{(0)}\right)$. This result implies that 

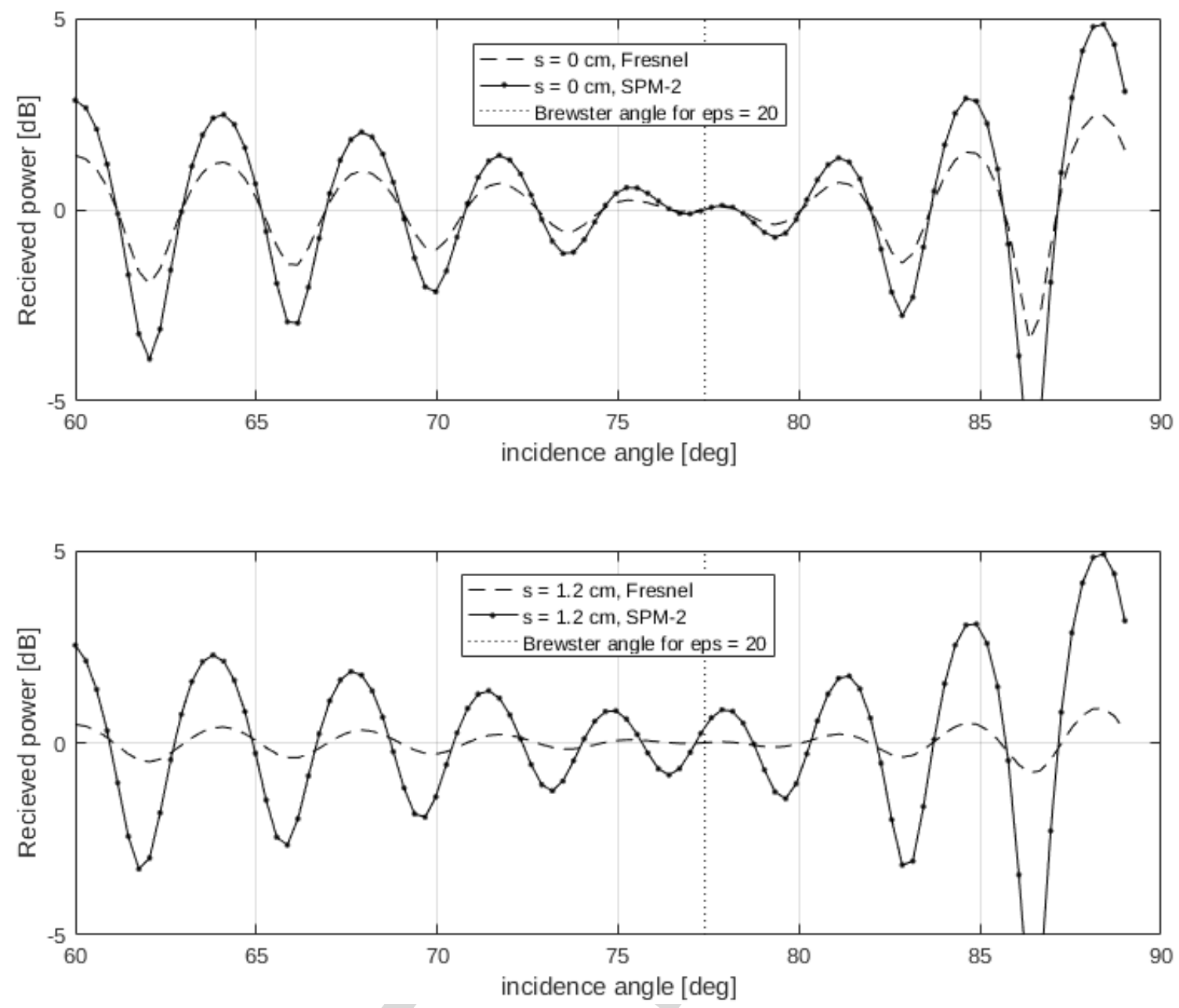

Fig. 3. (Top) Simulated IP as a function of the incidence angle according to two different models (modified Fresnel and SPM-2) for a low value of soil rms $s=0 \mathrm{~cm}$ (plane interface). (Bottom) Simulated IP as a function of the incidence angle according to two different models (modified Fresnel and SPM-2) for a typical value of soil rms $s=1.2 \mathrm{~cm}$ [20]. Results correspond to soil dielectric constant $\epsilon=20$, correlation length $l=10 \mathrm{~cm}$, and L-band operating frequency. The scattered angle is equal to the incidence angle $\theta_{s}=\theta_{i}$.

the SM retrieval based on simplified approaches (see [13]) will have problems in detecting the notch of the IP, since it may not be present. However, the simulations based on SPM-2 do predict a reduction in the amplitude of the ITP (that, however, could not correspond with the Brewster angle, see Fig. 1). This is important since typical agricultural soils (related to the "no tillage" practice) are characterized by mean values of $s$ of the order of $1 \mathrm{~cm} \mathrm{[17],} \mathrm{a} \mathrm{region} \mathrm{in} \mathrm{which} \mathrm{(according}$ to our simulations) the notch will not be present and only a minimum in the ITP will be observed.

Next, using typical values of bare soil rms roughness $s$ [17]-[20], the objective is now to test how the simulated IP changes as a function of the dielectric constant of the soil according to SPM-2 model. In Fig. 2, we simulated the vertically polarized IP as a function of the incidence angle for different values of $\epsilon$, keeping the value of $s$ fixed. It can be seen that no notch is present, but a minimum in the IP can be seen for all the range of dielectric constants. Moreover, the angle in which this minimum occurs varies with $\epsilon$, as expected from theory. However, for this typical value of agricultural soil roughness, the position of the minimum will be difficult to locate with simple techniques-in particular, for large values of $\epsilon$, in which the Brewster angle is known to saturate.

Finally, it is important to evaluate how the results proposed here differ from the standard approach in [13] and related works. Reference [13, eqs. (4)-(9)] presents the scattering model used to represent the observed signal in the antenna [as discussed in Section II, the final expression is qualitatively similar to the first term of the SPM2 developed here (5)]. Combining simulations based on the expressions developed in [13] and ours, in Fig. 3, the IPs for the two models are shown for comparison, considering two extreme values of the soil rms height $s(s=0 \mathrm{~cm}, s=1.2 \mathrm{~cm})$.

As seen, for low values of soil $\mathrm{rms}(s=0 \mathrm{~cm}$, a plane interface), both models predict a notch which coincides with the Brewster angle. For $s=1.2 \mathrm{~cm}$, according to the modified Fresnel model, the notch is still present and the overall effect of soil roughness is to reduce the IP amplitude. However, according to our simulations (SPM-2), the notch disappears, but a minimum in the IP is still present. This differential behavior between models is related to the fact that SPM-2 
includes multiple scattering effects, which are neglected in the modified Fresnel approach. Nevertheless, if soil roughness has low values $(s \sim 0.5 \mathrm{~cm})$, the notch will be present and standard techniques can be used successfully.

\section{CONCLUSION}

In this letter, a new approach to simulate the IP based on SPM-2 was proposed. We showed simulations that confirm the basic behavior of the observed signal (in particular of the "notch"), and its expected monotonic behavior as a function of soil dielectric constant. However, according to our simulations, the notch amplitude and position depend strongly on soil roughness, a behavior that, according to our knowledge, is not present in published models.

The IPT is promising since by measuring in the forward direction, it has low sensitivity requirements for the receptor. Moreover, by measuring the IP, no narrow angular pattern of the receptor antenna is required. A drawback to this technique is that it necessarily operates at low elevation angles (very large scattering angles) for which the Brewster angle is present. Moreover, it saturates quickly for large values of the dielectric constant. In addition to these known constraints, according to our simulations, soil roughness needs to be taken into account in the SM retrieval scheme. Finally, the results presented here are only valid for bare soils (a very atypical condition of agricultural soils). In future work, we expect to include the contribution of the vegetation to both signal scattering and attenuation in the computation of the interference pattern.

\section{REFERENCES}

[1] F. T. Ulaby, R. K. Moore, and A. K. Fung, Microwave Remote Sensing: Active and Passive. Radar Remote Sensing and Surface Scattering and Emission Theory, vol. 2. Reading, MA, USA: Addison-Wesley, 1982.

[2] L. Tsang and J. A. Kong, Scattering of Electromagnetic Waves, Advanced Topics, vol. 26. Hoboken, NJ, USA: Wiley, 2004.

[3] J. T. Johnson, "Third-order small-perturbation method for scattering from dielectric rough surfaces," J. Opt. Soc. Amer. A, vol. 16, no. 11, pp. 2720-2736, 1999.

[4] M. A. Demir and J. T. Johnson, "Fourth- and higher-order small-perturbation solution for scattering from dielectric rough surfaces," J. Opt. Soc. Amer. A, vol. 20, no. 12, pp. 2330-2337, 2003.

[5] M. A. Demir, J. T. Johnson, and T. J. Zajdel, "A study of the fourth-order small perturbation method for scattering from two-layer rough surfaces," IEEE Trans. Geosci. Remote Sens., vol. 50, no. 9, pp. 3374-3382, Sep. 2012.

[6] J. T. Johnson and J. D. Ouellette, "Polarization features in bistatic scattering from rough surfaces," IEEE Trans. Geosci. Remote Sens., vol. 52, no. 3, pp. 1616-1626, Mar. 2014.
[7] N. Pierdicca, L. Guerriero, R. Giusto, M. Brogioni, and A. Egido, "SAVERS: A simulator of GNSS reflections from bare and vegetated soils," IEEE Trans. Geosci. Remote Sens., vol. 52, no. 10 , pp. 6542-6554, Oct. 2014.

[8] A. Egido et al., "Global navigation satellite systems reflectometry as a remote sensing tool for agriculture," Remote Sens., vol. 4, no. 8, pp. 2356-2372, 2012.

[9] A. Egido et al., "Airborne GNSS-R polarimetric measurements for soil moisture and above-ground biomass estimation," IEEE J. Sel. Topics Appl. Earth Observ. Remote Sens., vol. 7, no. 5, pp. 1522-1532, May 2014.

[10] D. Masters, P. Axelrad, and S. Katzberg, "Initial results of land-reflected GPS bistatic radar measurements in SMEX02," Remote Sens. Environ. vol. 92, no. 4, pp. 507-520, Sep. 2004.

[11] S. J. Katzberg, O. Torres, M. S. Grant, and D. Masters, "Utilizing calibrated GPS reflected signals to estimate soil reflectivity and dielectric constant: Results from SMEX02," Remote Sens. Environ., vol. 100, no. 1, pp. 17-28, Jan. 2006.

[12] A. Kavak, G. Xu, and W. J. Vogel, "Gps multipath fade measurements to determine 1-band ground reflectivity properties," in Proc. 20th NASA Propag. Experimenters Meeting, 1996, pp. 257-261.

[13] N. Rodriguez-Alvarez et al., "Soil moisture retrieval using GNSS-R techniques: Experimental results over a bare soil field," IEEE Trans. Geosci. Remote Sens., vol. 47, no. 11, pp. 3616-3624, Nov. 2009.

[14] A. A. Arroyo et al., "Dual-polarization GNSS-R interference pattern technique for soil moisture mapping," IEEE J. Sel. Topics Appl. Earth Observat. Remote Sens., vol. 7, no. 5, pp. 1533-1544, May 2014

[15] A. Alonso-Arroyo et al., "Improving the accuracy of soil moisture retrievals using the phase difference of the dual-polarization GNSS-R interference patterns," IEEE Geosci. Remote Sens. Lett., vol. 11, no. 12, pp. 2090-2094, Dec. 2014.

[16] N. Rodríguez-Alvarez, "Contributions to earth observation using GNSSR opportunity signals," Ph.D. dissertation, Dept. Theory Commun., Univ. Politécnica Catalunya, Barcelona, Spain, 2011. Accessed: Oct. 4, 2015. [Online]. Available: http://www.tdx.cat/handle/10803/53636

[17] T. J. Jackson, H. McNairn, M. A. Weltz, B. Brisco, and R. Brown, "First order surface roughness correction of active microwave observations for estimating soil moisture," IEEE Trans. Geosci. Remote Sens., vol. 35, no. 4, pp. 1065-1069, Jul. 1997.

[18] M. W. J. Davidson, T. L. Toan, F. Mattia, G. Satalino, T. Manninen, and M. Borgeaud, "On the characterization of agricultural soil roughness for radar remote sensing studies," IEEE Trans. Geosci. Remote Sens., vol. 38, no. 2, pp. 630-640, Mar. 2000.

[19] M. W. J. Davidson et al., "Joint statistical properties of RMS height and correlation length derived from multisite 1-m roughness measurements," IEEE Trans. Geosci. Remote Sens., vol. 41, no. 7, pp. 1651-1658, Jul. 2003

[20] J. Álvarez-Mozos, J. Casalí, M. González-Audícana, and N. E. Verhoest, "Assessment of the operational applicability of RADARSAT-1 data for surface soil moisture estimation," IEEE Trans. Geosci. Remote Sens., vol. 44, no. 4, pp. 913-924, Apr. 2006.

[21] N. E. Verhoest, H. Lievens, W. Wagner, J. Álvarez-Mozos, M. S. Moran, and F. Mattia, "On the soil roughness parameterization problem in soil moisture retrieval of bare surfaces from synthetic aperture radar," Sensors, vol. 8, no. 7, pp. 4213-4248, 2008.

[22] M. E. Barber, F. M. Grings, J. Álvarez-Mozos, M. Piscitelli, P. A. Perna, and $\mathrm{H}$. Karszenbaum, "Effects of spatial sampling interval on roughness parameters and microwave backscatter over agricultural soil surfaces," Remote Sens., vol. 8, no. 6, p. 458, 2016.

[23] P. Chen et al., "Second-order small perturbation method for transmission from dielectric rough surfaces," Waves Random Complex Media, vol. 21, no. 4, pp. 668-689, 2011. 


\title{
Analysis of the Effect of Soil Roughness in the Forward-Scattering Interference Pattern Using Second-Order Small Perturbation Method Simulations
}

\author{
Mariano Franco ${ }^{\circledR}$, Emanuel More, Esteban Roitberg ${ }^{\circledR}$, Francisco Grings ${ }^{\circledR}$, Member, IEEE, \\ Estefanía Piegari, Vanesa Douna, and Pablo Perna
}

\begin{abstract}
Soil moisture (SM) is a key geophysical variable that can be estimated at regional scales using remote sensing techniques, by making use of the known relationship between soil reflectivity and the dielectric constant in the microwave regime. In this context, the exploitation of available illuminators of opportunity that currently emit large amounts of power at microwave frequencies (compared to typical synthetic aperture radar systems) is promising. Some published techniques estimate SM by analyzing the interference pattern (IP) between direct and reflected signal as measured by a single antenna (i.e., IP technique). In this letter, a new approach to simulate the IP is proposed, in which the soil roughness is modeled straightforwardly using the second-order small perturbation model. Results illustrate that the "notch" in the VV-polarization IP (related to the Brewster angle) can only be directly observed for very low values of soil rms roughness $(s<0.5 \mathrm{~cm})$. For typical values of soil roughness $(s \sim 1.2 \mathrm{~cm})$, the notch disappears and only a minimum in the IP is observed near the Brewster angle.
\end{abstract}

Index Terms-Electromagnetic and remote sensing, Global Navigation Satellite System data, microwave radiometry, surface and subsurface properties.

\section{INTRODUCTION}

A TYPICAL approach for estimating surface soil moisture (SM) at regional scales and high spatial resolution is based on the exploitation of the signal reflected from the soil in the microwave regime, using the well-known relationship between the dielectric constant and SM. However, the scattered signal by the surface is not only determined by SM but also surface properties as its rms height $(s)$ and the geometry of the incident wave. Then, in order to get a retrieval algorithm for the SM, it is essential to count with a coherent scattering model which physically relates the relevant parameters involved in the scattering process. A well-established theoretical model to relate the soil backscattering coefficient $\left(\sigma^{0}\right)$ with the soil dielectric constant $(\epsilon)$ and soil rms roughness $s$ is the small

Manuscript received July 23, 2018; revised October 22, 2018; accepted November 18, 2018. This work was supported by PICT Raíces 2014-0647. (Corresponding author: Mariano Franco.)

M. Franco, E. Roitberg, F. Grings, E. Piegari, V. Douna, and P. Perna are with the Instituto de Astronomía y Física del Espacio (CONICET-UBA), Pabellón IAFE, CABA, Buenos Aires 1428, Argentina (e-mail: mfranco@df.uba.ar; verderis@iafe.uba.ar).

E. More is with the Instituto de Altos Estudios Espaciales "Mario Gulich," CONAE, Córdoba 5187, Argentina.

Digital Object Identifier 10.1109/LGRS.2018.2882688 perturbation model (SPM) [1], [2], which has been successfully studied under several different conditions for the surface or the incident wave [3]-[6]. However, to directly invert the second-order SPM (SPM-2) in order to estimate $\epsilon, \sigma^{0}$ of the terrain at high resolution is required, a task that can only be accomplished by a synthetic aperture radar, a relatively expensive, and power-demanding instrument.

In this letter, illuminators of opportunity (IOO) present several operative advantages for estimating SM. First, IOO bistatic radar configurations do not require a dedicated transmitter, which significantly reduces implementation costs (in both power and overall mass). Second, at least at L-band $(\lambda \sim 25 \mathrm{~cm})$, there is evidence that soil forward scattering presents a similar sensitivity to SM than the backscattered signal [7]. Typical SM retrieval techniques based on IOOs rely on the measurement of the soil forward-scattering coefficient, which is related to SM through the dielectric constant. The main benefit of this approach is that the ratio between reflected and transmitted fields is a direct proxy of SM. However, this approach has several drawbacks. One of the most significant drawbacks is the very good antenna isolation required to separate direct and reflected components (reflected component is usually -10 to $-20 \mathrm{~dB}$ below direct component, see [8]-[11]). Therefore, a very low level of crosstalk between antennas can be tolerated.

As an alternative, for IOOs characterized by sufficiently long pulses, the interference pattern technique (IPT) was proposed [12]. This technique is based on measuring the vertically polarized component of the received signal at the antenna. This "vertically polarized interference pattern (IP)" is analyzed in order to find a minimum ("notch"), which according to theory should correspond to the Brewster angle, which is itself related to the surface dielectric constant and SM [12]. Therefore, this technique has the advantage of requiring only one antenna and thus it does not rely on signal separation, but on the signal coherent sum. This technique has been successfully implemented and validated using fieldwork in [8], [11], and [13].

However, the analysis presented in [13] and its subsequents [14]-[16] present a key limitation: they are based on a simplified model for soil specular scattering. This means that it is assumed that soil specular scattering can be modeled 
using a plane interface multiplied by a roughness term to analyze the relationship between the angle in which the notch is found and then the dielectric constant of the soil. Therefore, the position and the presence of the notch itself are based on a simplified scattering model, which does take into account soil roughness but does not include multiple scattering effects on the surface. Hence, this analysis could be improved by using a more advanced scattering model.

In this letter, we implemented the SPM-2 [2]-[4] for simulating the vertically polarized IP expected in the antenna as a function of both geometric and dielectric soil properties (soil dielectric constant and roughness). We developed a model that computes the coherent sum between the electric field scattered by the soil and the direct component emitted by the IOO as a function of surface characteristics, assuming incident circular polarization and received vertical polarization. In the analysis, we show that the SPM-2 predicts the notch for very smooth soils. In this condition, the notch expected monotonic behavior as a function of soil dielectric constant is also observed. However, the existence of the notch depends strongly on soil roughness due to multiple scattering processes that take place in the illuminated surface at this frequency.

This letter is organized as follows. In Section II, we introduce the scattering model and a solution for the proposed geometry. In Section III, we present key simulation results that show the expected vertically polarized IP for different soil conditions and system configurations. In particular, the position and amplitude of the ITP minimum are studied. Finally, in Section IV, we present some conclusions derived from the analysis.

\section{SCATtering Model: Second-ORdeR Small Perturbation Method}

The SPM is based on the hypothesis that soil surface has a small $\mathrm{rms}$ height $(s)$ with respect to the incident wavelength $(\lambda)$, specifically that $2 \pi s / \lambda \ll 0.3$ [1], [2]. Usually, the SPM is used even in the limit $s \sim 0.05 \lambda$, producing satisfactory results (see [3]-[6]). For our case $(\lambda=25 \mathrm{~cm})$, the condition to be satisfied is $s<1.25 \mathrm{~cm}$, which is a reasonable assumption since for bare agricultural soils, typical values of rms height are $s \sim 1 \mathrm{~cm} \mathrm{[17]-[22].} \mathrm{This} \mathrm{approach}$ is based on proposing that the scattered and transmitted fields above and below the surface satisfy the boundary conditions of the Maxwell equations. The scattered and transmitted fields are written as a power series expansion in terms of the surface height $z$, where each term accounts for different scattering mechanisms on the surface. The zeroth-order term shows the specular reflection, the first-order term gives a single scattering behavior, and the second order represents a multiplescattering process where the incident wave after the first interaction with the surface goes to a second point, reflects again and finally propagates to the free space. At this point, it is important to remark that at the second order, the SPM verifies energy conservation [23]. Thus, we are proposing a physically based scattering theory that includes both surface roughness and energy conservation effects.

We implemented the SPM up to the second order to compute the IP observed in the antenna. We deal with circular polarization (GPS systems); therefore, the incident wave and reflected wave (RV) can be decomposed in terms of horizontal and vertical polarizations. As the antenna only measures vertical polarization, we must compute the vertical polarization of the RV, which has two components: VV and HV. The first one is due to a vertical incident polarization which remains in the same polarization after it interacts with the surface; the latter is due to the cross polarization effect, by which a wave with horizontal polarization changes its state after it scatters with the surface. It is well known that the cross polarization is due to multiple scattering effects [2]-[5], and thus it requires the development of the SPM up to the second order.

Based on SPM, the scattered field is expressed as [2]-[4]

$$
\mathbf{E}_{s} \approx \int \frac{d^{2} k}{(2 \pi)^{2}} e^{\imath \mathbf{k} \cdot \mathbf{r}} \sum_{n}\left[\alpha^{(n)} \hat{h}_{s}+\beta^{(n)} \hat{v}_{s}\right]
$$

being $\hat{h}_{s}$ and $\hat{v}_{s}$ the horizontal and vertical polarizations of the scattered field. Up to the second order, the amplitude of the vertical polarization mode is

$$
\begin{aligned}
\beta(\mathbf{k}) \approx \beta^{(0)} \delta\left(\mathbf{k}-\mathbf{k}_{i}\right) & +\beta^{(1)} Z(\mathbf{k}) \\
& +\int d^{2} k^{\prime} \beta^{(2)}\left(\mathbf{k}, \mathbf{k}^{\prime}\right) Z\left(\mathbf{k}-\mathbf{k}^{\prime}\right)
\end{aligned}
$$

with a similar expression for the amplitude $\alpha$ (for a detailed development of these coefficients, see [2]-[4]). First- and second-order terms depend on the Fourier transform of the surface, $Z(\mathbf{k})$. We see that the second-order term shows a process of multiple scattering through the integration of an auxiliary mode in $Z$. We have a set of parameters $\left\{\alpha^{(n)}, \beta^{(n)}\right\}$ for the TE mode and a different set for the TM mode.

Considering that we are interested in finding the scattered field in vertical polarization from an incident wave with circular polarization, we must compute the dot product between the expression (1) and $\hat{v}_{s}$. Thus, we will have the VV contribution given by the set of TE and the HV contribution given by the set of TM. For simplicity, we call them $\xi^{(n)}$. In Section II-A, we will compute the IP due to the incident and scattered waves.

$$
\begin{aligned}
\left\langle E_{T} E_{T}^{*}\right\rangle= & \frac{1}{E_{i}^{2}}\left\langle\left(E_{i}+E_{S}\right) \cdot\left(E_{i}^{*}+E_{s}^{*}\right)\right\rangle \\
\left\langle E_{T} E_{T}^{*}\right\rangle \approx & \left\langle\left\{e^{\imath \mathbf{k}_{i} \cdot \mathbf{r}_{A}}+\int \frac{d^{2} k}{(2 \pi)^{2}} e^{l \mathbf{k} \cdot \mathbf{r}_{A}}\left[\xi^{(0)} \delta\left(\mathbf{k}-\mathbf{k}_{i}\right)+\xi^{(1)} Z(\mathbf{k})+\int \frac{d^{2} k^{\prime}}{(2 \pi)^{2}} \xi^{(2)}\left(\mathbf{k}, \mathbf{k}^{\prime}\right) Z\left(\mathbf{k}-\mathbf{k}^{\prime}\right)\right]\right\}\right. \\
& \left.\times\left\{e^{-l \mathbf{k}_{i} \cdot \mathbf{r}_{A}}+\int \frac{d^{2} k}{(2 \pi)^{2}} e^{-l \mathbf{k} \cdot \mathbf{r}_{A}}\left[\xi^{(0)} \delta\left(\mathbf{k}-\mathbf{k}_{i}\right)+\xi^{(1)} Z(\mathbf{k})+\int \frac{d^{2} k^{\prime}}{(2 \pi)^{2}} \xi^{(2)}\left(\mathbf{k}, \mathbf{k}^{\prime}\right) Z\left(\mathbf{k}-\mathbf{k}^{\prime}\right)\right]\right\}\right\rangle
\end{aligned}
$$




\section{A. Interference Pattern}

With the goal of computing the IP received by the antenna, we must compute the mean value of the total field measured by it. As we have a stochastic RV, due to the scattering from a random rough surface, the IP will depend on statistical properties of the surface (i.e., roughness spectrum $W(\mathbf{k})$, rms height $s$, and correlation length $l$ ). Therefore, we must compute the mean power of the total intensity normalized to the incident field amplitude, which is shown in (3) shown at the bottom of the previous page.

Here, $\mathbf{r}_{A}=\left(x_{A}, z_{A}\right)$ indicates the antenna position. To perform the mean value, we only need to know that the surface can be modeled as a stationary and isotropic random process, with a Gaussian distribution of heights and zero mean. This kind of stochastic process has the following properties: $\langle Z(\mathbf{k})\rangle=0$ and $\left\langle Z(\mathbf{k}) Z^{*}\left(\mathbf{k}^{\prime}\right)\right\rangle=W\left(\mathbf{k}-\mathbf{k}^{\prime}\right)$, where the last quantity is the roughness spectrum of the surface, which we consider Gaussian, namely, $W(\mathbf{k})=$ $\left(s^{2} l^{2} / 4 \pi\right) \exp \left[-l^{2} \mathbf{k}^{2} / 4\right]$. By using these properties, the intensity pattern that emerges from a straightforward but tedious calculation results in

$$
\begin{aligned}
\left\langle E_{T} E_{T}^{*}\right\rangle \approx & +2 \xi^{(0)} \cos \left(2 k_{s z} z_{A}\right)+\left[\xi^{(0)}\right]^{2} \\
& +2 \Re\left[e^{\imath 2 k_{s z} z A} \int d^{2} k^{\prime} \xi^{(2)} W\left(\mathbf{k}_{i}-\mathbf{k}^{\prime}\right)\right] \\
& +\left|\xi^{(1)}\left(\mathbf{k}_{i}\right)\right|^{2} \int d^{2} k^{\prime} W\left(\mathbf{k}^{\prime}\right) \\
& +2 \xi^{(0)} \int d^{2} k^{\prime} \Re\left[\xi^{(2)}\left(\mathbf{k}_{i}, \mathbf{k}^{\prime}\right) W\left(\mathbf{k}_{i}-\mathbf{k}^{\prime}\right)\right] .
\end{aligned}
$$

The first line shown in (4) is similar (but not exactly equal) to the expression derived in [13], where the power intensity is proportional to the Fresnel reflection coefficient (here noted by $\left.\xi^{(0)}\right)$ multiplied by a Gaussian roughness factor and to the phase difference between the incident and RV (i.e., $\Delta \phi=$ $\left.2 k_{s z} z_{A}\right)$

$$
\begin{aligned}
\left\langle E_{T} E_{T}^{*}\right\rangle & \approx 1+2 \xi^{(0)} \cos \left(2 k_{s z} z_{A}\right)+\left[\xi^{(0)}\right]^{2} \\
& =\left|1+\xi^{(0)} e^{t 2 k_{s z} z_{A}}\right|^{2} .
\end{aligned}
$$

As we are using a scattering method based on small perturbations, the above-mentioned term does not include any roughness factor, because it is the zeroth-order solution. The surface roughness appears naturally in the following terms, which are proportional to the roughness spectrum $W(\mathbf{k})$. Moreover, for typical agricultural soils $(s \approx 1 \mathrm{~cm})$, both terms, $\xi^{(1)}$ and $\xi^{(2)}$, are relevant because they take into account the multiple scattering effects produced on the surface.

In Section III, we use (4) to simulate the behavior of the IP measured in the antenna.

\section{Simulated Results}

In Section II, we obtained the theoretical expression of the IP, which depends on the illumination geometry and surface parameters (geometric and dielectric). In Fig. 1, the behavior of the signal measured in the antenna is shown as a function of the incidence angle for several values of soil rms height $s$ : values near $1 \mathrm{~cm}$ are typical for no-till soil management [18], [19], [22]; other less typical management

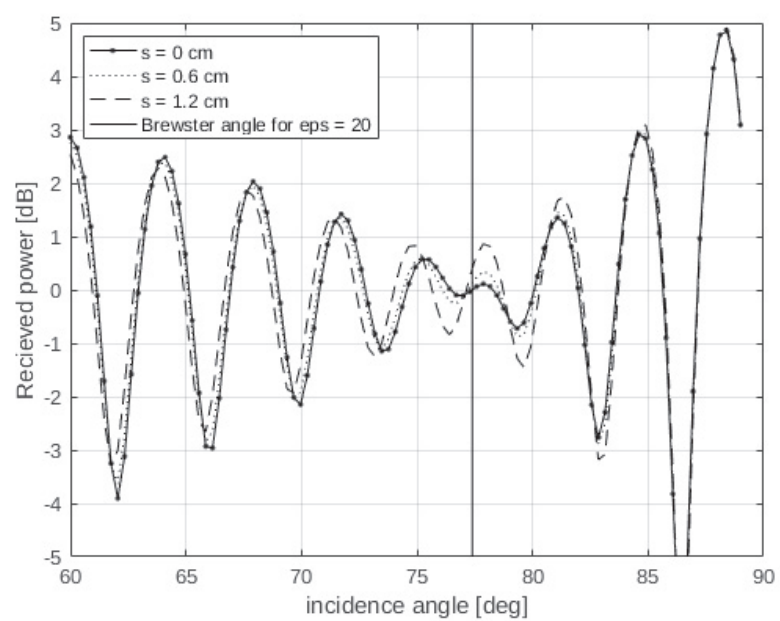

Fig. 1. Simulated IP as a function of the incidence angle for different values of the surface rms height $s$. Results correspond to L-band, i.e., $\lambda=25 \mathrm{~cm}$, correlation length $l=10 \mathrm{~cm}$, dielectric constant $\epsilon=20$ (which is related to a medium SM value), and the scattered angle is equal to the incidence angle $\theta_{s}=\theta_{i}$.

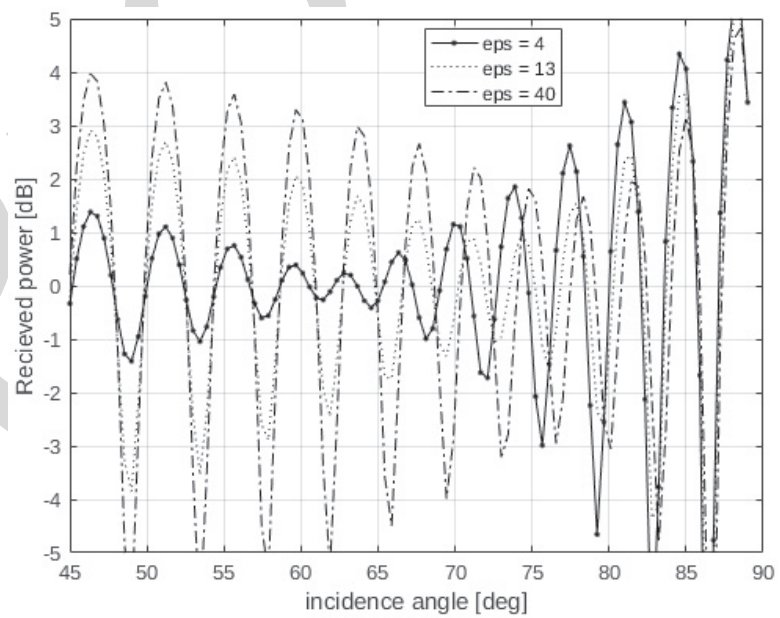

Fig. 2. Simulated IP as a function of the incident angle for different values of soils dielectric constant $\epsilon$. Results correspond to soil roughness $s=1 \mathrm{~cm}$ (typical of agricultural soils), correlation length $l=10 \mathrm{~cm}$, and L-band operating frequency. The scattered angle is equal to the incidence angle $\theta_{s}=\theta_{i}$.

(e.g., harrow or roll) lead to values $\sim 0.5 \mathrm{~cm} \mathrm{[20],} \mathrm{[21].}$ In addition, in plowed fields, the soil rms height can reach values in the range of $2.5-4 \mathrm{~cm} \mathrm{[20],} \mathrm{[21],} \mathrm{for} \mathrm{which} \mathrm{notch}$ detection will be more difficult, as we will show in Fig. 1. Finally, in order to plot the IP, the antenna is located at $z_{A}=2 \mathrm{~m}$ (or $8 \lambda$ in the worst case, which satisfies the far-field condition).

As expected for a flat surface $(s=0 \mathrm{~cm})$, a notch corresponding to the Brewster angle is present for large values of the incidence angle, which is in agreement with (5) and the results presented in [13]. As $s$ increases the notch starts to vanish, almost disappearing for relatively large values of the roughness $(s=1.2 \mathrm{~cm} \sim 0.05 \lambda)$. This is related to the fact that for a very rough surface, the diffuse scattering (proportional to the factors $\xi^{(1)}$ and $\xi^{(2)}$, which take into account the effects of multiple scattering) becomes more relevant than the specular reflection (just proportional to $\xi^{(0)}$ ). This result implies that 

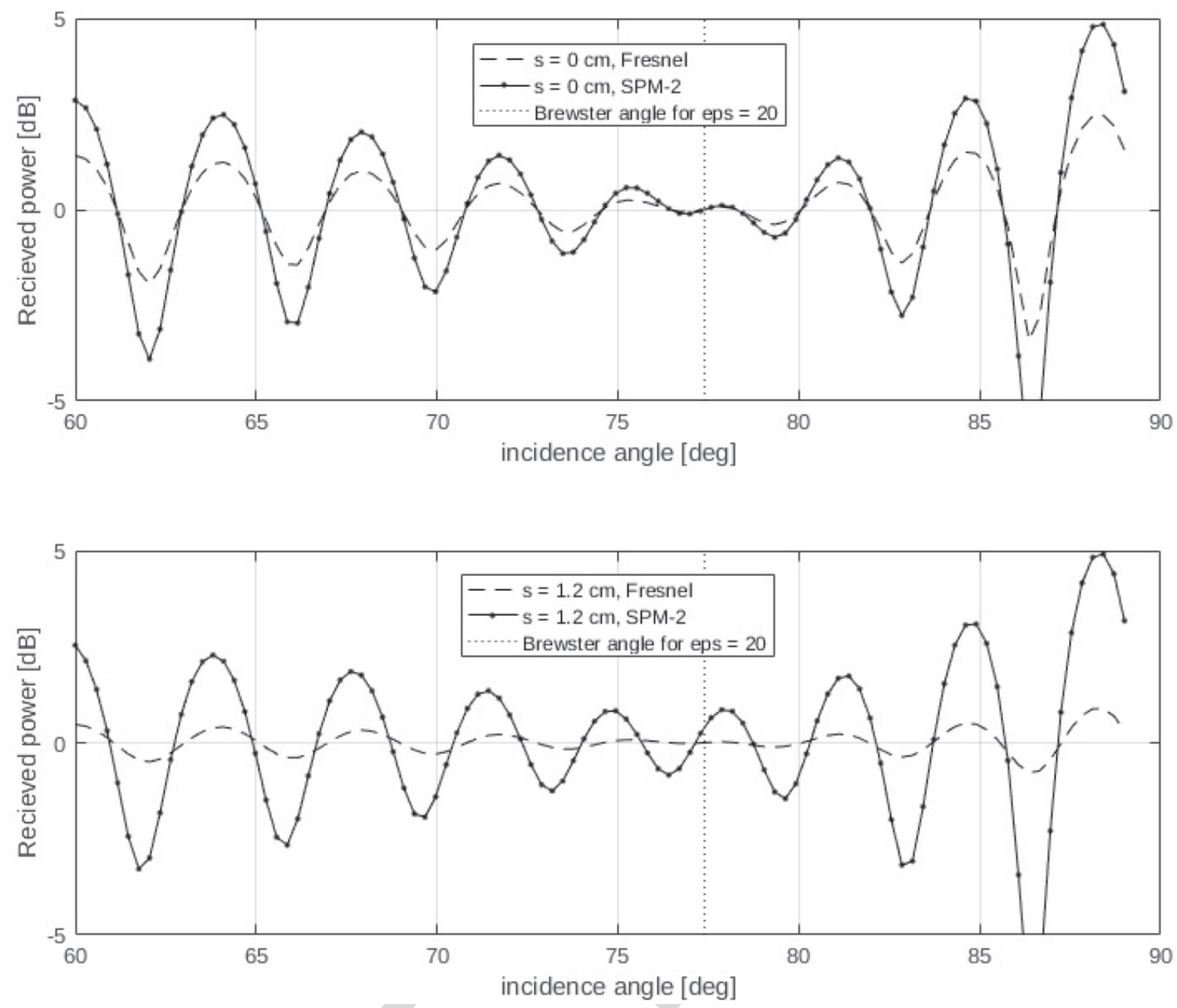

Fig. 3. (Top) Simulated IP as a function of the incidence angle according to two different models (modified Fresnel and SPM-2) for a low value of soil rms $s=0 \mathrm{~cm}$ (plane interface). (Bottom) Simulated IP as a function of the incidence angle according to two different models (modified Fresnel and SPM-2) for a typical value of soil rms $s=1.2 \mathrm{~cm}$ [20]. Results correspond to soil dielectric constant $\epsilon=20$, correlation length $l=10 \mathrm{~cm}$, and L-band operating frequency. The scattered angle is equal to the incidence angle $\theta_{s}=\theta_{i}$.

the SM retrieval based on simplified approaches (see [13]) will have problems in detecting the notch of the IP, since it may not be present. However, the simulations based on SPM-2 do predict a reduction in the amplitude of the ITP (that, however, could not correspond with the Brewster angle, see Fig. 1). This is important since typical agricultural soils (related to the "no tillage" practice) are characterized by mean values of $s$ of the order of $1 \mathrm{~cm} \mathrm{[17],} \mathrm{a} \mathrm{region} \mathrm{in} \mathrm{which} \mathrm{(according}$ to our simulations) the notch will not be present and only a minimum in the ITP will be observed.

Next, using typical values of bare soil rms roughness $s$ [17]-[20], the objective is now to test how the simulated IP changes as a function of the dielectric constant of the soil according to SPM-2 model. In Fig. 2, we simulated the vertically polarized IP as a function of the incidence angle for different values of $\epsilon$, keeping the value of $s$ fixed. It can be seen that no notch is present, but a minimum in the IP can be seen for all the range of dielectric constants. Moreover, the angle in which this minimum occurs varies with $\epsilon$, as expected from theory. However, for this typical value of agricultural soil roughness, the position of the minimum will be difficult to locate with simple techniques-in particular, for large values of $\epsilon$, in which the Brewster angle is known to saturate.

Finally, it is important to evaluate how the results proposed here differ from the standard approach in [13] and related works. Reference [13, eqs. (4)-(9)] presents the scattering model used to represent the observed signal in the antenna [as discussed in Section II, the final expression is qualitatively similar to the first term of the SPM2 developed here (5)]. Combining simulations based on the expressions developed in [13] and ours, in Fig. 3, the IPs for the two models are shown for comparison, considering two extreme values of the soil rms height $s(s=0 \mathrm{~cm}, s=1.2 \mathrm{~cm})$.

As seen, for low values of soil $\mathrm{rms}(s=0 \mathrm{~cm}$, a plane interface), both models predict a notch which coincides with the Brewster angle. For $s=1.2 \mathrm{~cm}$, according to the modified Fresnel model, the notch is still present and the overall effect of soil roughness is to reduce the IP amplitude. However, according to our simulations (SPM-2), the notch disappears, but a minimum in the IP is still present. This differential behavior between models is related to the fact that SPM-2 
includes multiple scattering effects, which are neglected in the modified Fresnel approach. Nevertheless, if soil roughness has low values $(s \sim 0.5 \mathrm{~cm})$, the notch will be present and standard techniques can be used successfully.

\section{CONCLUSION}

In this letter, a new approach to simulate the IP based on SPM-2 was proposed. We showed simulations that confirm the basic behavior of the observed signal (in particular of the "notch"), and its expected monotonic behavior as a function of soil dielectric constant. However, according to our simulations, the notch amplitude and position depend strongly on soil roughness, a behavior that, according to our knowledge, is not present in published models.

The IPT is promising since by measuring in the forward direction, it has low sensitivity requirements for the receptor. Moreover, by measuring the IP, no narrow angular pattern of the receptor antenna is required. A drawback to this technique is that it necessarily operates at low elevation angles (very large scattering angles) for which the Brewster angle is present. Moreover, it saturates quickly for large values of the dielectric constant. In addition to these known constraints, according to our simulations, soil roughness needs to be taken into account in the SM retrieval scheme. Finally, the results presented here are only valid for bare soils (a very atypical condition of agricultural soils). In future work, we expect to include the contribution of the vegetation to both signal scattering and attenuation in the computation of the interference pattern.

\section{REFERENCES}

[1] F. T. Ulaby, R. K. Moore, and A. K. Fung, Microwave Remote Sensing: Active and Passive. Radar Remote Sensing and Surface Scattering and Emission Theory, vol. 2. Reading, MA, USA: Addison-Wesley, 1982.

[2] L. Tsang and J. A. Kong, Scattering of Electromagnetic Waves, Advanced Topics, vol. 26. Hoboken, NJ, USA: Wiley, 2004.

[3] J. T. Johnson, "Third-order small-perturbation method for scattering from dielectric rough surfaces," J. Opt. Soc. Amer. A, vol. 16, no. 11, pp. 2720-2736, 1999.

[4] M. A. Demir and J. T. Johnson, "Fourth- and higher-order small-perturbation solution for scattering from dielectric rough surfaces," J. Opt. Soc. Amer. A, vol. 20, no. 12, pp. 2330-2337, 2003.

[5] M. A. Demir, J. T. Johnson, and T. J. Zajdel, "A study of the fourth-order small perturbation method for scattering from two-layer rough surfaces," IEEE Trans. Geosci. Remote Sens., vol. 50, no. 9, pp. 3374-3382, Sep. 2012.

[6] J. T. Johnson and J. D. Ouellette, "Polarization features in bistatic scattering from rough surfaces," IEEE Trans. Geosci. Remote Sens., vol. 52, no. 3, pp. 1616-1626, Mar. 2014.
[7] N. Pierdicca, L. Guerriero, R. Giusto, M. Brogioni, and A. Egido, "SAVERS: A simulator of GNSS reflections from bare and vegetated soils," IEEE Trans. Geosci. Remote Sens., vol. 52, no. 10 , pp. 6542-6554, Oct. 2014.

[8] A. Egido et al., "Global navigation satellite systems reflectometry as a remote sensing tool for agriculture," Remote Sens., vol. 4, no. 8, pp. 2356-2372, 2012.

[9] A. Egido et al., "Airborne GNSS-R polarimetric measurements for soil moisture and above-ground biomass estimation," IEEE J. Sel. Topics Appl. Earth Observ. Remote Sens., vol. 7, no. 5, pp. 1522-1532, May 2014.

[10] D. Masters, P. Axelrad, and S. Katzberg, "Initial results of land-reflected GPS bistatic radar measurements in SMEX02," Remote Sens. Environ. vol. 92, no. 4, pp. 507-520, Sep. 2004.

[11] S. J. Katzberg, O. Torres, M. S. Grant, and D. Masters, "Utilizing calibrated GPS reflected signals to estimate soil reflectivity and dielectric constant: Results from SMEX02," Remote Sens. Environ., vol. 100, no. 1, pp. 17-28, Jan. 2006.

[12] A. Kavak, G. Xu, and W. J. Vogel, "Gps multipath fade measurements to determine 1-band ground reflectivity properties," in Proc. 20th NASA Propag. Experimenters Meeting, 1996, pp. 257-261.

[13] N. Rodriguez-Alvarez et al., "Soil moisture retrieval using GNSS-R techniques: Experimental results over a bare soil field," IEEE Trans. Geosci. Remote Sens., vol. 47, no. 11, pp. 3616-3624, Nov. 2009.

[14] A. A. Arroyo et al., "Dual-polarization GNSS-R interference pattern technique for soil moisture mapping," IEEE J. Sel. Topics Appl. Earth Observat. Remote Sens., vol. 7, no. 5, pp. 1533-1544, May 2014

[15] A. Alonso-Arroyo et al., "Improving the accuracy of soil moisture retrievals using the phase difference of the dual-polarization GNSS-R interference patterns," IEEE Geosci. Remote Sens. Lett., vol. 11, no. 12, pp. 2090-2094, Dec. 2014.

[16] N. Rodríguez-Alvarez, "Contributions to earth observation using GNSSR opportunity signals," Ph.D. dissertation, Dept. Theory Commun., Univ. Politécnica Catalunya, Barcelona, Spain, 2011. Accessed: Oct. 4, 2015. [Online]. Available: http://www.tdx.cat/handle/10803/53636

[17] T. J. Jackson, H. McNairn, M. A. Weltz, B. Brisco, and R. Brown, "First order surface roughness correction of active microwave observations for estimating soil moisture," IEEE Trans. Geosci. Remote Sens., vol. 35, no. 4, pp. 1065-1069, Jul. 1997.

[18] M. W. J. Davidson, T. L. Toan, F. Mattia, G. Satalino, T. Manninen, and M. Borgeaud, "On the characterization of agricultural soil roughness for radar remote sensing studies," IEEE Trans. Geosci. Remote Sens., vol. 38, no. 2, pp. 630-640, Mar. 2000.

[19] M. W. J. Davidson et al., "Joint statistical properties of RMS height and correlation length derived from multisite 1-m roughness measurements," IEEE Trans. Geosci. Remote Sens., vol. 41, no. 7, pp. 1651-1658, Jul. 2003

[20] J. Álvarez-Mozos, J. Casalí, M. González-Audícana, and N. E. Verhoest, "Assessment of the operational applicability of RADARSAT-1 data for surface soil moisture estimation," IEEE Trans. Geosci. Remote Sens., vol. 44, no. 4, pp. 913-924, Apr. 2006.

[21] N. E. Verhoest, H. Lievens, W. Wagner, J. Álvarez-Mozos, M. S. Moran, and F. Mattia, "On the soil roughness parameterization problem in soil moisture retrieval of bare surfaces from synthetic aperture radar," Sensors, vol. 8, no. 7, pp. 4213-4248, 2008.

[22] M. E. Barber, F. M. Grings, J. Álvarez-Mozos, M. Piscitelli, P. A. Perna, and $\mathrm{H}$. Karszenbaum, "Effects of spatial sampling interval on roughness parameters and microwave backscatter over agricultural soil surfaces," Remote Sens., vol. 8, no. 6, p. 458, 2016.

[23] P. Chen et al., "Second-order small perturbation method for transmission from dielectric rough surfaces," Waves Random Complex Media, vol. 21, no. 4, pp. 668-689, 2011. 